\title{
ON FUNCTIONS THAT APPROXIMATE RELATIONS
}

\author{
GERALD BEER
}

\begin{abstract}
Let $X$ be a metric space and let $Y$ be a separable metric space. Suppose $R$ is a relation in $X \times Y$. The following are equivalent: (a) for each $\varepsilon>0$ there exists $f$ : $X \rightarrow Y$ such that the Hausdorff distance from $f$ to $R$ is at most $\varepsilon$; (b) the domain of $R$ is a dense subset of $X$, and for each isolated point $x$ of the domain the vertical section of $R$ at $x$ is a singleton; (c) for each $\varepsilon>0$ there exists $f: X \rightarrow Y$ of Baire class one such that the Hausdorff distance from $f$ to $R$ is at most $\varepsilon$.
\end{abstract}

Let $\left\langle X, d_{X}\right\rangle$ and $\left\langle Y, d_{Y}\right\rangle$ be metric spaces. By a relation $R$ in $X \times Y$ we mean a nonempty subset of the product. Let us make $X \times Y$ a metric space by defining the distance $\rho$ between points $\left(x_{1}, y_{1}\right)$ and $\left(x_{2}, y_{2}\right)$ in the product by

$$
\rho\left[\left(x_{1}, y_{1}\right),\left(x_{2}, y_{2}\right)\right]=\max \left\{d_{X}\left(x_{1}, x_{2}\right), d_{Y}\left(y_{1}, y_{2}\right)\right\} \text {. }
$$

A function $f: X \rightarrow Y$ will be said [3] to E-approximate $R$ if each point in $f$ has $\rho$-distance at most $\varepsilon$ from some point in $R$, and each point of $R$ has $\rho$-distance at most $\varepsilon$ from some point in $f$. Alternatively, $f$ can be called [2] an $\varepsilon$-approximate selection for $R$, although this terminology has been used differently by Michael [7] and Deutsch and Kenderov [4]. More formally, if $f$ is an $\varepsilon$-approximate selection for $R$, then $f$ has Hausdorff distance at most $\varepsilon$ from $R$. We now pause to describe this notion.

Let $W$ be a metric space. For each point $w$ in $W$ let $S_{\varepsilon}[w]$ denote the open ball of radius $\varepsilon$ with center $w$ in $W$. If $C \subset W$ denote $\cup_{w \in C} S_{\varepsilon}[w]$ by $S_{\varepsilon}[C]$. If $K$ is another set in $W$ and there exists $\varepsilon>0$ for which both $S_{\varepsilon}[C] \supset K$ and $S_{\varepsilon}[K] \supset C$, then the Hausdorff distance $\delta$ between $C$ and $K$ is given by

$$
\delta[C, K]=\inf \left\{\varepsilon: S_{\varepsilon}[C] \supset K \text { and } S_{\varepsilon}[K] \supset C\right\} .
$$

If no such $\varepsilon$ exists, we write $\delta[C, K]=\infty$. Further information on this notion of distance can be found in Aubin [1], Kuratowski [6], or Nadler [9]. Now if $\delta$ denotes Hausdorff distance in $X \times Y$ as induced by $\rho$ and $R$ is a nonempty subset of $X \times Y$ and $f: X \rightarrow Y$, then the symbol $\delta[f, R]$ makes sense, and it is clear that (i) if $f$ $\varepsilon$-approximates $R$, then $\delta[f, R] \leqslant \varepsilon$; (ii) if $\delta[f, R] \leqslant \varepsilon$ then $f \theta$-approximates $R$ for each $\theta>\varepsilon$.

The main purpose of this note is to characterize for arbitrary $X$ and separable $Y$ those relations in $X \times Y$ that admit for each $\varepsilon>0$ a Borel $\varepsilon$-approximate selection. We shall in fact show that the existence for each $\varepsilon>0$ of an $\varepsilon$-approximate selection

Received by the editors August 18, 1982.

1980 Mathematics Subject Classification. Primary 41A65, 54C65, 54B20; Secondary 54C50.

Key words and phrases. Approximate selection, Hausdorff metric, functions of Baire class one. 
(Borel measurable or not) for the relation implies the existence for each $\varepsilon>0$ of a Baire class one $\varepsilon$-approximate selection.

Definition. Let $X$ and $Y$ be metric spaces. A function $f: X \rightarrow Y$ is said to be of Baire class $\alpha<\Omega$ if for each open subset $G$ of $Y$ the set $f^{-1}(G)$ is of additive class $\alpha$ in $X$.

In particular, $f: X \rightarrow Y$ is of Baire class one if the inverse image of each open subset of $Y$ is an $F_{\sigma}$ subset of $X$. For a thorough discussion of such functions, the reader should consult Kuratowski [6], where the functions of Baire class $\alpha$ are called $B$-measurable of class $\alpha$. We need two results from this source, which we state as lemmas. The first is not deep; the second is a serious theorem of Montgomery [8].

Lemma A. Let $X$ and $Y$ be metric spaces. Suppose $\left\{A_{i}: i \in Z^{+}\right\}$is a collection of sets each of additive class $\alpha$ with union $X$. Suppose $f: X \rightarrow Y$ and for each $i \in Z^{+}$the restriction of $f$ to $A_{i}$ is of Baire class $\alpha$. Then $f$ is of Baire class $\alpha$.

Lemma B. Let $X$ be a metric space and let $F \subset X$. Suppose for each $x \in X$ there exists an open neighborhood $V_{x}$ of $x$ such that $F \cap V_{x}$ is of additive class $\alpha$. Then $F$ itself is of additive class $\alpha$.

Since open sets in a metric space are $F_{\sigma}$ sets, the phrase " $F \cap V_{x}$ is of additive class $\alpha$ " used in Lemma B is unambigous: subsets of $V_{x}$ that are of additive class $\alpha$ with respect to the relative topology on $V_{x}$ are precisely those that are of additive class $\alpha$ with respect to the topology on $X$. In the sequel we shall use the following notation for the domain and vertical section at $x$ of a relation $R$ in $X \times Y$ :

$$
\operatorname{Dom}(R)=\{x: \text { for some } y,(x, y) \in R\}, \quad R(x)=\{y:(x, y) \in R\} .
$$

THEOREM 1. Let $X$ be a metric space and $Y$ a separable metric space. Suppose $R$ is a relation in $X \times Y$. The following are equivalent.

(a) For each $\varepsilon>0$ there exists $f: X \rightarrow Y$ such that $\delta[f, R] \leqslant \varepsilon$.

(b) The domain of $R$ is a dense subset of $X$, and for each isolated point $x$ of the domain the section $R(x)$ is a singleton.

(c) For each $\varepsilon>0$ there exists $f: X \rightarrow Y$ of Baire class one such that $\delta[f, R] \leqslant \varepsilon$.

Proof. (a) $\rightarrow$ (b). Suppose that $\overline{\operatorname{Dom}(R)} \neq X$. Then there exists $x \in X$ and $\varepsilon>0$ such that $S_{\varepsilon}[x] \cap \operatorname{Dom}(R)=\varnothing$. It follows that if $f: X \rightarrow Y$ is arbitrary, then $f \not \subset S_{\varepsilon}[R]$, whence $\delta[f, R] \geqslant \varepsilon$. Suppose now that $\overline{\operatorname{Dom}(R)}=X$, but for some isolated point $x$ of $\operatorname{Dom}(R)$ the section $R(x)$ contains two distinct points $y_{1}$ and $y_{2}$ of $Y$. Since $x$ must be an isolated point of $X$, there exists $\varepsilon>0$ such that both $d_{Y}\left(y_{1}, y_{2}\right)>\varepsilon$ and $S_{\varepsilon}[x]=\{x\}$. Hence if $f: X \rightarrow Y$ satisfies $\delta[f, R]<\varepsilon / 2$ we must simultaneously have $d_{Y}\left(f(x), y_{1}\right)<\varepsilon / 2$ and $d_{Y}\left(f(x), y_{2}\right)<\varepsilon / 2$, an impossibility.

(b) $\rightarrow$ (c). If $X$ has no limit points, then $\operatorname{Dom}(R)=X$ and each vertical section of $R$ is a singleton. Thus, $R$ is a continuous function, and there is nothing to prove. Otherwise, let $\theta=\varepsilon / 2$ and let $L$ denote the set of limit points of $X$. Consider the family $\Omega$ of subsets $S$ of $L$ with the following property: for each $\{x, z\} \subset S$, $d_{X}(x, z) \geqslant \theta$. If $\Omega$ is partially ordered by inclusion, then by Zorn's lemma $\Omega$ has a maximal member, say, $\left\{x_{i}: i \in I\right\}$, and it easily follows that $L \subset \cup_{i \in I} S_{\theta}\left[x_{i}\right]$. Let 
$C=\left\{y_{n}: n \in Z^{+}\right\}$be a countable dense subset of $Y$, and for each $i \in I$ choose $y_{n(i)} \in C$ whose distance from $\cup\left\{R(x): x \in S_{\theta}\left[x_{i}\right] \cap \operatorname{Dom}(R)\right\}$ is less than $\theta$. Since $W=\cup_{i \in I} S_{\theta}\left[x_{i}\right]$ as a subspace of $X$ is paracompact and regular, there is an open refinement $\left\{V_{\lambda}: \lambda \in \Lambda\right\}$ of the cover $\left\{S_{\theta}\left[x_{i}\right]: i \in I\right\}$ of $W$ such that $\left\{\bar{V}_{\lambda}\right.$ : $\lambda \in \Lambda\}$ is a locally finite (closed) refinement of $\left\{S_{\theta}\left[x_{i}\right]: i \in I\right\}$. Let $E=$ $\cup_{i \in I} S_{\theta / 3}\left[x_{i}\right]$. We first define a Baire class one function on this open subspace of $X$. Let $i \in I$ be arbitrary. Since $x_{i}$ is a limit point of $X$, there is a sequence $\left\{x_{n i}\right\}$ of distinct points in $S_{\theta / 3}\left[x_{i}\right]$ convergent to $x_{i}$. We define $h_{i}: S_{\theta / 3}\left[x_{i}\right] \rightarrow Y$ as follows: let $h_{i}$ map $\left\{x_{n i}: n \in Z^{+}\right\}$onto a dense subset of $\cup\left\{R(x): x \in S_{\theta}\left[x_{i}\right] \cap \operatorname{Dom}(R)\right\}$, and let $h_{i}$ assign to each remaining point of $S_{\theta / 3}\left[x_{i}\right]$ the point $y_{n(i)}$. Functions with countable domains are automatically of Baire class one; so $h_{i} \mid\left\{x_{n i}: n \in Z^{+}\right\}$and $h_{i} \mid S_{\theta / 3}\left[x_{i}\right]-\left\{x_{n i}: n \in Z^{+}\right\}$are both of Baire class one. Since the set $\left\{x_{n i}\right.$ : $\left.n \in Z^{+}\right\}$and its complement in $S_{\theta / 3}\left[x_{i}\right]$ are both $F_{\sigma}$ sets, Lemma A implies that $h_{i}$ itself is of Baire class one. Now set $h=\cup_{i \in I} h_{i}$. By the construction of $\left\{x_{i}: i \in I\right\}$, it is clear that $h$ is a well-defined function from $E$ to $Y$. Since the inverse image of each open set under $h$ is locally an $F_{\sigma}$ set, Lemma B ensures that $h$ is of Baire class one.

We next define a Baire class one function on $W-E$. For each $\lambda \in \Lambda$ choose $i(\lambda) \in I$ such that $\bar{V}_{\lambda} \subset S_{\theta}\left[x_{i(\lambda)}\right]$. For each $x \in W-E$ let $g(x)=y_{n(i(\lambda))}$ where $n(i(\lambda))$ is the smallest integer such that $x \in \bar{V}_{\lambda}$. We claim that for each $m \in Z^{+}$the set $g^{-1}\left(\left\{y_{1}, y_{2}, \ldots, y_{m}\right\}\right)$ is a relatively closed subset of $W-E$. To see this let $\left\{w_{k}\right\}$ be a sequence in $g^{-1}\left(\left\{y_{1}, y_{2}, \ldots, y_{m}\right\}\right)$ convergent to some point $w$ of $W-E$. Since $\left\{\bar{V}_{\lambda}: \lambda \in \Lambda\right\}$ is locally finite there exist indices $\left\{\lambda_{1}, \lambda_{2}, \ldots, \lambda_{p}\right\} \subset \Lambda$ and an integer $K$ such that for each $k>K,\left\{\lambda: \lambda \in \Lambda\right.$ and $\left.w_{k} \in \bar{V}_{\lambda}\right\} \subset\left\{\lambda_{1}, \lambda_{2}, \ldots, \lambda_{p}\right\}$. Now for each $k>K$ there exists $\lambda(k) \in\left\{\lambda_{1}, \lambda_{2}, \ldots, \lambda_{p}\right\}$ such that $w_{k} \in \bar{V}_{\lambda(k)}$ and $n(i(\lambda(k)))$ $\leqslant m$. Since $\left\{\lambda_{1}, \lambda_{2}, \ldots, \lambda_{p}\right\}$ is finite, there exists $\lambda \in\left\{\lambda_{1}, \lambda_{2}, \ldots, \lambda_{p}\right\}$ for which $w \in \bar{V}_{\lambda}$ and $n(i(\lambda)) \leqslant m$. This establishes the claim. Since the intersection of a closed set with an open set is an $F_{\sigma}$ set, for each $m \geqslant 2$,

$$
g^{-1}\left(\left\{y_{m}\right\}\right)=g^{-1}\left(\left\{y_{1}, \ldots, y_{m}\right\}\right)-g^{-1}\left(\left\{y_{1}, \ldots, y_{m-1}\right\}\right)
$$

is a relatively $F_{\sigma}$ subset of $W-E$. Hence for each open set $G$ of $Y$ the set $g^{-1}(G)=g^{-1}(C \cap G)$ is a relatively $F_{\sigma}$ subset of $W-E$, and it follows that $g$ : $W-E \rightarrow Y$ is of Baire class one.

On $X-W$ the relation $R$ reduces to a continuous function. Since the sets $E$, $W-E$, and $X-W$ are each $F_{\sigma}$ subsets of $X$, by Lemma A the function $f: X \rightarrow Y$ defined by

$$
f(x)= \begin{cases}h(x) & \text { if } x \in E \\ g(x) & \text { if } x \in W-E \\ R(x) & \text { if } x \in X-W\end{cases}
$$

is of Baire class one. It remains to show that $\delta[f, R] \leqslant \varepsilon=2 \theta$. We first show that each point in $f$ is within $\varepsilon$ of some point in $R$. If $x \in X-W$ then $(x, f(x)) \in R$. If $x \in W-E$ then there exists $i \in I$ such that $x \in S_{\theta}\left[x_{i}\right]$ and $f(x)=y_{n(i)}$. However, by the definition of $y_{n(i)}$ there exists a point $x_{i}^{*}$ in $S_{\theta}\left[x_{i}\right]$ and a point $y \in R\left(x_{i}^{*}\right)$ for 
which $d_{Y}\left(y, y_{n(i)}\right)<\theta$. It follows that $\rho\left[(x, f(x)),\left(x_{i}^{*}, y\right)\right]<\max \{2 \theta, \theta\}=\varepsilon$. Finally, if $x \in E$ then there exists $i \in I$ such that $d_{X}\left(x, x_{i}\right)<\theta / 3$; moreover, $f(x)$ is either $y_{n(i)}$ or a point in $\bigcup\left\{R(z): z \in S_{\theta}\left[x_{i}\right] \cap \operatorname{Dom}(R)\right\}$. In either case $(x, f(x))$ has $\rho$-distance less than $\varepsilon$ from some point $\left(x_{i}^{*}, y\right)$, where $x_{i}^{*} \in S_{\theta}\left[x_{i}\right]$ and $y \in R\left(x_{i}^{*}\right)$. We now must show that each point of $R$ is within $\varepsilon$ of some point of $f$. If $x \in X-W$ then $R(x)$ is a singleton and $R(x)=f(x)$. Next let $x \in W \cap \operatorname{Dom}(R)$ and choose $y \in R(x)$. There exists $i \in I$ such that $x \in S_{\theta}\left[x_{i}\right]$. Recall, however, that $\left\{f\left(x_{n i}\right): n \in Z^{+}\right\}$is dense in $\cup\left\{R(z): z \in S_{\theta}\left[x_{i}\right] \cap \operatorname{Dom}(R)\right\}$, so there exists $n \in Z^{+}$for which $d_{Y}\left(f\left(x_{n i}\right), y\right)<\varepsilon$. Again, it is clear that $\rho\left[(x, y),\left(x_{n i}, f\left(x_{n i}\right)\right)\right]<$ $\varepsilon$, and this portion of the proof is complete.

(c) $\rightarrow$ (a). Obvious.

Theorem 1 fails without the separability assumption on $Y$.

EXAMPLE 1. Let $X$ be the rationals, viewed as a subspace of the line with the usual topology, and let $Y$ be an uncountable set with the discrete metric. Let $R=X \times Y$. Now each $f: X \rightarrow Y$ has a countable range, and it follows from the definition of the metric $\rho$ on $X \times Y$ that $\delta[f, R]=1$.

Following Michael we could call $f: X \rightarrow Y$ an $\varepsilon$-approximate selection for a relation $R$ with domain $X$ if, for each $x$ in $X, f(x) \in S_{\varepsilon}[R(x)]$. The existence of Baire class one approximate selections in this context would seem to rest on some continuity requirement on the map $x \rightarrow R(x)$. For example, the property of almost lower semicontinuity, due to Deutsch and Kenderov [4], is sufficient [2]: for each $x$ in $X$ there exists a neighborhood $V_{x}$ of $x$ such that $\cap\left\{S_{\varepsilon}[R(w)]: w \in V_{x}\right\}$ is nonempty.

EXAmple 2. Let $X=Y=[0,1]$ and let $B$ be a non-Borel set in the interval. Let $R \subset X \times Y$ be the characteristic function of the set $B$. Then if $f: X \rightarrow Y$ is a $\frac{1}{3}$-approximate selection for $R$ (in the sense of Michael), then $f^{-1}\left(\left(\frac{1}{2}, 1\right]\right)=B$, a non-Borel set. Thus, $R$ admits no Borel $\frac{1}{3}$-approximate selection.

Continuous approximate selections, either in our sense or that of Michael, can be obtained for certain well-behaved relations with convex vertical sections. A recent example: if $X$ is paracompact and $Y$ is a normed linear space, then those relations with domain $X$ that admit for each $\varepsilon>0$ a continuous $\varepsilon$-approximate selection in the sense of Michael are precisely those that are almost lower semicontinuous [4]. Invariably, such approximations are constructed by piecing together continuous functions defined locally via a partition of unity [5, p. 170] to yield a globally defined continuous function that is close to the relation. We close by showing that locally defined Baire class $\alpha$ functions are subject to such an amalgamation, provided $X$ is metric and $Y$ is a second countable topological vector space.

Theorem 2. Let $X$ be a metric space and let $Y$ be a second countable topological vector space. Let $\left\{U_{\lambda}: \lambda \in \Lambda\right\}$ be a locally finite open cover of $X$ and let $\left\{p_{\lambda}(\cdot)\right.$ : $\lambda \in \Lambda\}$ be a partition of unity subordinated to the cover. Suppose for each $\lambda \in \Lambda$ the function $f_{\lambda}: U_{\lambda} \rightarrow Y$ is of Baire class $\alpha$. Then $f: X \rightarrow Y$ defined by $f(x)=$ $\Sigma_{\lambda \in \Lambda} p_{\lambda}(x) f_{\lambda}(x)$ is of Baire class $\alpha$.

Proof. Fix $x$ in $X$ and let $V_{x}$ be an open neighborhood of $x$ that meets only finitely many members of the open cover, say $\left\{U_{\lambda_{1}}, \ldots, U_{\lambda_{n}}\right\}$. By Lemma B we need 
only show that $f \mid V_{x}$ is of Baire class $\alpha$. Now for each $z$ in $V_{x}$ we have $f(z)=$ $\sum_{i=1}^{n} p_{\lambda_{i}}(z) f_{\lambda_{i}}(z)$. Since the restriction of each function of Baire class $\alpha$ is of Baire class $\alpha$ on its restricted domain, to show that $f \mid V_{x}$ is of Baire class $\alpha$ it suffices to show that if $h_{1}: X \rightarrow Y$ and $h_{2}: X \rightarrow Y$ are of Baire class $\alpha$ and $p$ is a real valued continuous function on $X$, then both $p h_{1}$ and $h_{1}+h_{2}$ are of Baire class $\alpha$. We prove the former statement, leaving the latter to the reader. Let $\left\{G_{i}: i \in Z^{+}\right\}$and $\left\{U_{i}\right.$ : $\left.i \in Z^{+}\right\}$be bases for the topologies on $Y$ and the line, respectively. Consider $\phi$ : $X \rightarrow Y \times R$ defined by $\phi(x)=\left(h_{1}(x), p(x)\right)$. Since $\phi^{-1}\left(G_{i} \times U_{j}\right)=h_{1}^{-1}\left(G_{i}\right) \cap$ $p^{-1}\left(U_{j}\right)$ and the sets of additive class $\alpha$ contain the open sets and are closed under finite intersections and countable unions, the second countability of $Y \times R$ implies $\phi^{-1}(G)$ is of additive class $\alpha$ for each open set $G$ in the product. Since $\psi: Y \times R \rightarrow Y$ defined by $\psi(y, \theta)=\theta y$ is continuous, $p h_{1}=\psi \circ \phi$ is of Baire class $\alpha$.

It is important to note that Theorem 2 cannot be used to piece together locally Borel functions to obtain a globally Borel function. Using the well-known example of Szpilrajn-Marczewski [6] of a non-Borel set in a metric space that is nevertheless locally Borel, a counterexample can be easily constructed. The details are left to the reader.

\section{REFERENCES}

1. J. P. Aubin, Applied abstract analysis, Wiley, New York, 1977.

2. G. Beer, On a theorem of Deutsch and Kenderov (submitted).

3. A. Cellina, $A$ further result on the approximation of set valued mappings, Atti Accad. Naz. Lincei Rend. Cl. Sci. Fis. Mat. Natur. (8) 48 (1970), 412-416.

4. F. Deutsch and P. Kenderov, Continuous selections and approximate selections for set-valued mappings and applications to metric projections, SIAM J. Math. Anal. 14 (1983), 185-194.

5. J. Dugundji, Topologv, Allyn and Bacon, Boston, Mass., 1966.

6. K. Kuratowski, Topology, Vol. 1, Academic Prèss, New York, 1966.

7. E. Michael, Selected selection theorems, Amer. Math. Monthly 63 (1956), 233-237.

8. D. Montgomery, Nonseparable metric spaces, Fund. Math. 25 (1935), 527-533.

9. S. Nadler, Hyperspaces of sets, Dekker, New York, 1978.

Department of Mathematics, California State University, los Angeles, Los Angeles, CaliFORNIA 90032 